Apidologie, 1974, 4 (4), 317-329.

\title{
RASTERELEKTRONENMIKROSKOPISCHE BEOBACHTUNGEN AN POLLENKÖRNERN WICHTIGER BRASILIANISCHER BIENENPFLANZEN
}

Observations sur le pollen de quelques plantes mellifères importantes du Brésil au moyen du microscope électronique à balayage

\author{
Ortrud Monika BARTH*
}

Instituto Oswaldo Cruz, Rio de Janeiro/Brasilien

SUMMARY

SCANNING ELECTRON MICROSCOPICAL OBSERVATIONS OF POLLEN
GRAINS FROM IMPORTANT BRAZILIAN HONEY PLANTS

In order to better understanding the fine structure of their pollen grains, eight important brazilian honey plant species producing unifloral honeys have been studied by scanning electron microscopy. The superficial structure of polyads from Mimosoideae and monads from Borreria verticillata and Eucalyptus robusta have been specially studied and described.

\section{ZUSAMMENFASSUNG}

Es wurden Pollenkörner von acht Sortenhonig liefernden Bienenpflanzen im Rasterelektronenmikroskop morphologisch untersucht, um ihre Strukturfeinheiten besser charakterisieren zu können. Besonders an Mimosoiden-Polyaden und Monaden von Borreria verticillata und Eucalyptus robusta konnten die bisher noch unklaren Oberfächenstrukturen genauestens erkannt und beschrieben werden.

* Mit Unterstiutzung des Conselho Nacional de Pesquisas. 


\section{I. - EINLEITUNG}

Als Ergänzung vorausgegangener lichtoptischer Untersuchungen an brasilianischen Honigen und Trachtpflanzen (BARTH 1969 u. 1970) wurden Pollenkörner von Sortenhonigen im Rasterelektronenmikroskop näher untersucht. Nach bisherigen Ergebnissen handelt es sich vorerst um acht Arten, da die brasilianische Bienenflora bisher nur ungenügend untersucht wurde. Palynologische Beschreibungen dieser Pollenkörner sowie der zugehörigen Honigproben wurden bereits näher von BARTH (1970) durchgeführt.

\section{II. - MATERIAL UND METHODEN}

Bei den für die Untersuchungen im Rasterelektronenmikroskop bestimmten Pollenarten handelt es sich um solche aus getrockneten Blüten, deren Anthereninhalt, suspendiert in einer Alkohol-Äther-Lösung, abzentrifugiert wurde. Ein Tropfen des Sedimentes wurde auf einem Glasträger gut eingetrocknet, mit Gold kegelförmig bedampft und in einem Stereoscan Scanning Electron Microscope 96113-2A, Cambridge, bei 10 oder 30 KV untersucht. Andere Präparate wurden vor der Goldbedampfung senkrecht kurz mit einer dünnen Kohleschicht überzogen und an einem JSM-U3 Scanning-Microscope, Jeol, bei $6 \mathrm{KV}$ photographiert.

Dank gebührt dem Departamento de Metalurgia da COPPE, UFRJ, welches die Bedampfung der Objekte und die rasterelektronenmikroskopischen Untersuchungen ermöglichte, sowie der Firma (Jeol do Brasil ", welche einige Aufnahmen unserer Präparate an ihrem Gerät im Instituto de Pesquisas Radioativas, Belo Horizonte, MG, vornahm.

\section{III. - ERGEBNISSE}

\section{Leguminosae-Mimosoideae}

1. Mimosa scabrella Benth. (Fig. 1-3)

Lichtoptisch gesehen, handelt es sich bei dieser Art um kleine Pollentetraden $(10,5 \times 8,5 \mu)$ mit glatter Oberfläche und durchsichtiger, farbloser Exine. Die elektronenmikroskopischen Untersuchungen ergaben jedoch eine deutlich granuläre Struktur der Sexine, wobei die Oberflächengranula im Durchschnitt $0,3-0,5 \mu$ groß sind. Die Ränder der einzelnen Pollenkörner sind im allgemeinen glatt, besonders über den Poren ist die Sexine meistens nicht unterbrochen.

Viele Arten dieser Gattung weisen solche kleinen Pollentetraden auf. Inwiefern sie als Pollen-oder Nektarpflanzen zu betrachten sind, steht noch nicht für alle fest. Bei den bekanntesten Arten dieses Pollentyps, z.B. $M$. pudica, $M$. sensitiva und $M$. veloziella, handelt es sich vorerst um Pollenlieferanten. Honige mit einem Anteil von mehr als $98 \%$ dieser Tetraden sind stets gelblich hell und klar, sehr wohlschmeckend und kristallisieren nicht aus. 
2. Mimosa caesalpiniaefolia Benth. (Fig. 4 und 5)

Beim Pollentyp dieser Art handelt es sich um Doppeltetraden $(12,5 \times 10 \mu)$ mit, lichtoptisch gesehen, glatter, durchsichtiger Exine. Elektronenoptisch ist die Oberfläche granulär, die Granula haben einen mittleren Durchmesser von $0,4 \mu$. Die Ränder der einzelnen Pollenkörner sind hier weniger glatt als bei $M$. scabrella. Wie schon lichtoptisch beobachtet, sind die Anstoßlinien je zwei zueinander gehörender Tetraden gegen einander versetzt, so daß die Verbindungslinien zweier Pollenkörner stets auf die Seitenlinie eines Pollenkornes der anderen Tetrade stoßen.

Honige dieses Pollentyps sind pollenärmer als die des $M$. scabrella- Typs, gelblich durchsichtig, wohlschmeckend und nicht kristallisierend.

\section{Piptadenia moniliformis Benth. (Fig. 6 und 7)}

Wieder handelt es sich um Gruppen von je acht Pollenkörnern, jedoch ungleichmässig angeordnet, so daß die Tetraden nicht von einander abgegrenzt sind. Die Gruppen sind etwas grösser als bei der vorhergehenden Art $(18 \times 13 \mu)$ mit, lichtoptisch gesehen, glatter, durchsichtiger Exine. Elektronenoptisch gesehen, ist die Pollenoberfläche jedoch unregelmäßig strukturiert, eher gewellt als granulär, mit abgesetzten glatteren Randflächen.

Es sind stets pollenarme Honige von bräunlich durchsichtiger Farbe, sehr wohlschmeckend und nicht kristallisierend.

\section{Rutaceae}

Citrus sinensis Osbeck (Fig. 8-12)

Mittelgroße $(28 \times 30 \mu)$, 4-colporate, reticulate Pollenkörner. Die Lumina sind klein, aber sehr tief, meistens rundlich, mit glatten Rändern, von breiten Muri getrennt und einigermaßen gleichmäßig über die ganze Pollenober fläche verteilt.

Citrus-Honige sind pollenarm, glänzend hellbraun und durchsichtig und kristallisieren nur teilweise aus. Ihres aromatischen Geschmackes wegen werden sie allgemein bevorzugt.

\section{Myrtaceae}

Eucalyptus robusta Sm. (Fig. 13 und 14)

Parasyncolpate Pollenkörner $(16 \times 21 \mu)$ mit dünnen, lichtoptisch oft undeutlich abgesetzten Apocolpien und glatter, durchsichtiger Exine. Elektro- 
nenmikroskopisch gesehen, erscheinen die Mesocolpien- und Apocolpienoberflächen leicht gewellt, dagegen sind die Colpenränder vollkommen glatt.

Eucalyptus-Honige sind pollenreich, braun bis rotbraun und kristallisieren selten und nur teilweise aus. Thres scharfen, aromatischen Geschmackes wegen sind sie nicht so beliebt wie die vorhergehend besprochenen Honigarten.

\section{Rubiaceae}

Borreria verticillata G. F. W. Mey. (Fig. 15 und 16)

Kleine $(21 \times 23 \mu)$, rundliche Pollenkörner, $(6-) 7(-8)$-colporat, mit lichtoptisch schwer zu bestimmender Oberflächenstruktur vom L.O. - Typ. Elektronenoptisch gesehen, handelt es sich dabei um sehr kleine Stacheln, die gleichmäßig verteilt einer glatten Oberfläche aufsitzen. Die Colpen sind sehr kurz und weisen keine besonders strukturierten Randfächen auf.

Honige dieser Art sind einigermaßen pollenreich, meistens dunkelbraun, kristallisieren teilweise aus und haben einen etwas scharfen, unangenehmen Geschmack.

\section{Labiatae}

Hyptis umbrosa Salzm. (Fig. 17-20)

6- colpate, reticulate Pollenkörner mittlerer Größe $(35 \times 36 \times 32 \mu)$. Colpen und Mesocolpien sind untereinander gleich. Die Abstufung an den fein ausgezackten Colpenrändern ist auf die Nexine zurückzuführen. Teile der Sexine reichen bis in die Mitte der Colpen hinein. Die Lumina sind kleiner und runder an den Polen, länglich an den Mesocolpien, sehr tief; auf ihrem Grund sind kleine Bacula wahrnehmbar.

Es sind pollenarme, braune Honige, die nicht auskristallisieren.

\section{Compositae}

Vernonia scorpioides Pers. (Fig. 21)

Mittelgrosse $(30 \times 33,5 \mu$, ohne Stacheln) bis grosse Pollenkörner, 3-colporat, mit einer rauhen, von grossen, langen, Bacula aufsitzenden Stacheln besetzten Oberfläche. Die Colpen liegen versteckt, bei nicht hydratierten Körnern fast unsichtbar zwischen den Stacheln verborgen. 
Reine Vernonia - Honige sind nicht sehr pollenreich, hellgelb bis hellbraun und kristallisieren meistens vollkommen aus; sie haben einen stark aromatischen, angenehmen Geschmack.

Eingegangen im Juni 1973.

Reçu pour publication en juin 1973.

\section{RÉSUME}

L'auteur a étudié au microscope électronique à balayage le pollen de huit plantes mellifères importantes du Brésil. Cette étude a permis de mieux comprendre des détails de structure difficilement observables au microscope optique. Les polyades de Mimosées ainsi que les monades de Borreria verticillata et Eucalyptus robusta ont révélé des structures de surface qui, jusqu'ici, n’avaient pas pu être clairement reconnues et qu'il a été possible de décrire.

\section{LITERAT UR}

Barth O. M., 1969, Pollenspektren einiger brasilianischer Bienenhonige. Z. Bienenforsch. $9(9), 410-419$.

Barth O. M., 1970, Análise microscópica de algumas amostras de mel. 1 - Pólen Dominante. An. Acad. brasil. Ciênc. 42 (2), 352-366. 
PLANCHE I.

Le trait clair correspond à $2 \mu$.

Fig. 1 à 3. - Mimosa scabrella

Fig. 4et 5. - Mimosa caesalpiniaefolia

Fic. 6. - Piptadenia moniliformis

TAFEL I.

Die weissen Segmente in den Figuren entsprechen stets $2 \mu$.

Fig. 1-3. - Mimosa scabrella

Fig. 4 und 5. - Mimosa caesalpiniaefolia

FIG. 6. - Piptadenia moniliformis 

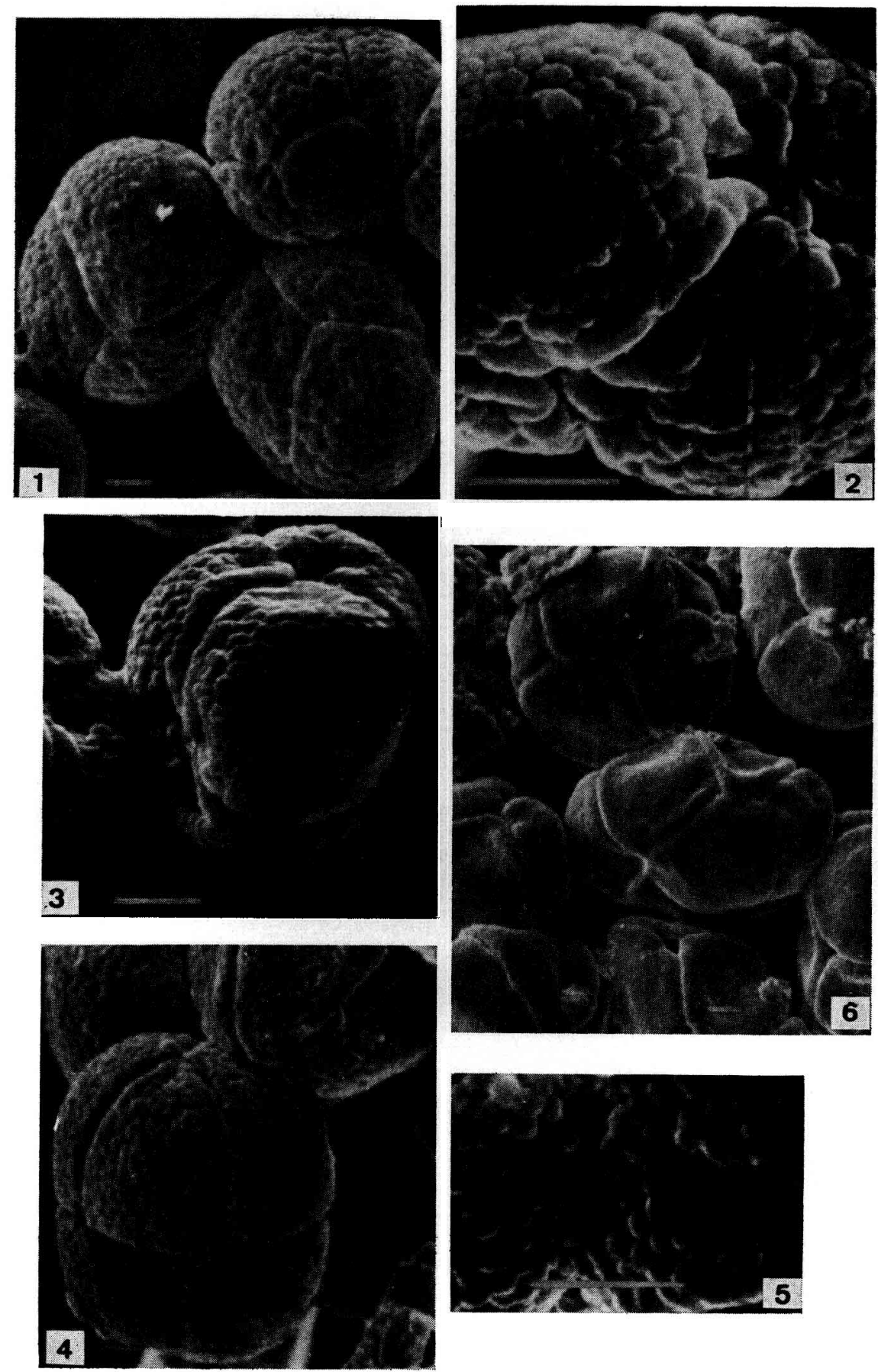

Ortrud Monika BaRth 


\section{PLANCHE II.}

Le trait clair correspond à $2 \mu$.

Fig. 7. - Piptadenia moniliformis

FIg. 8 à 12. - Citrus sinensis

TAFEL II.

Die weissen Segmente in den Figuren entsprechen stets $2 \mu$.

Fig. 7. - Piptadenia moinliformis

FIG. 8-12. - Citrus sinensis 

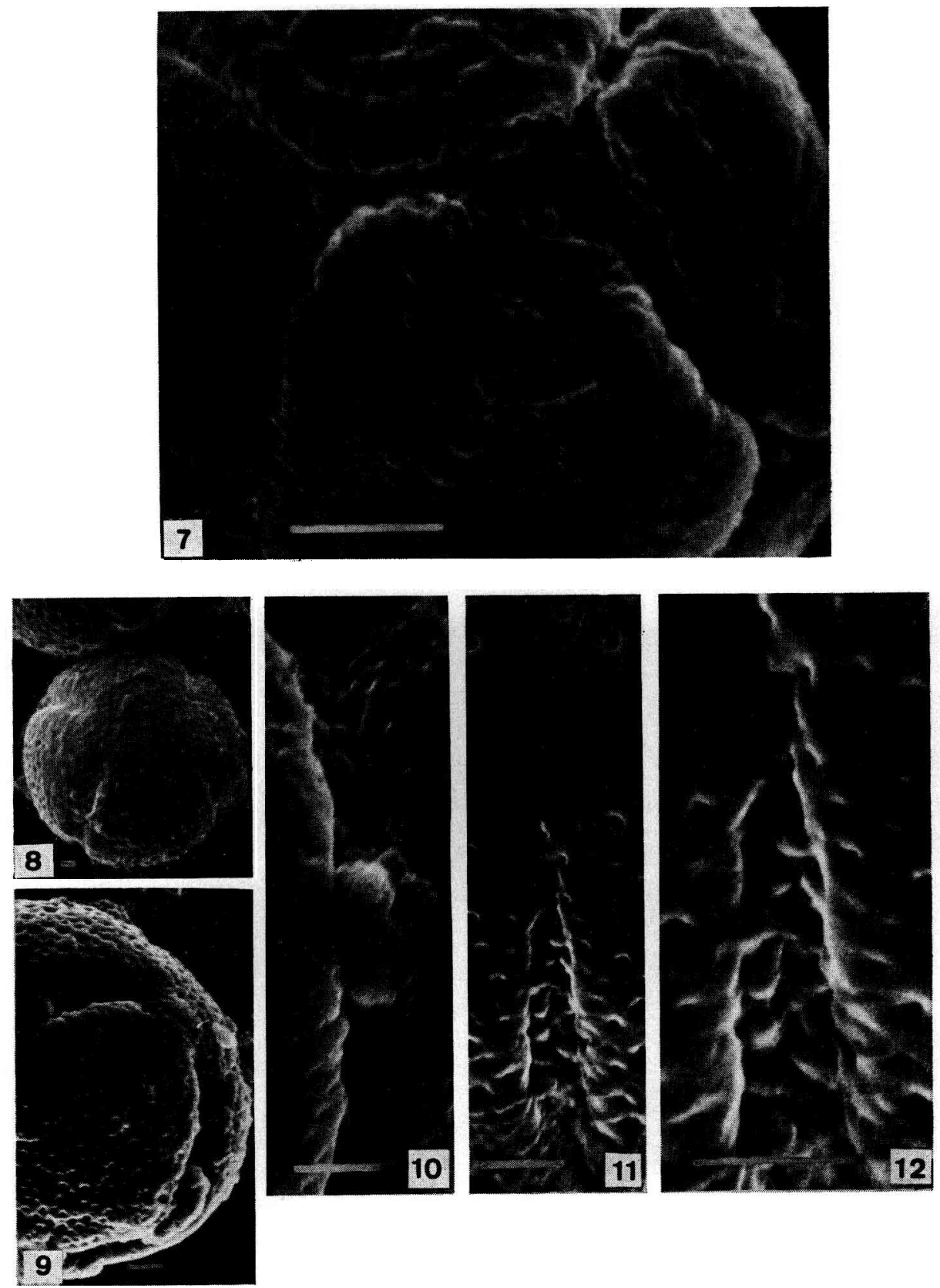

Ortrud Monika BaRTH 


\section{PLANCHE III.}

Le trait clair correspond à $2 \mu$. Fig. 13 et 14. - Eucalyptus robusta Fig. 15 et 16. - Borreria verticillata

\section{TAFEL III.}

Die weissen Segmente in den Figuren entsprechen ${ }_{-}^{\circ}$ stets $2^{-} \mu$.

Fig. 13 und 14. - Eucalyptus robusta

FIg. 15 und 16. - Borreria verticillata 
Apidologie, 1973, 4 (4).
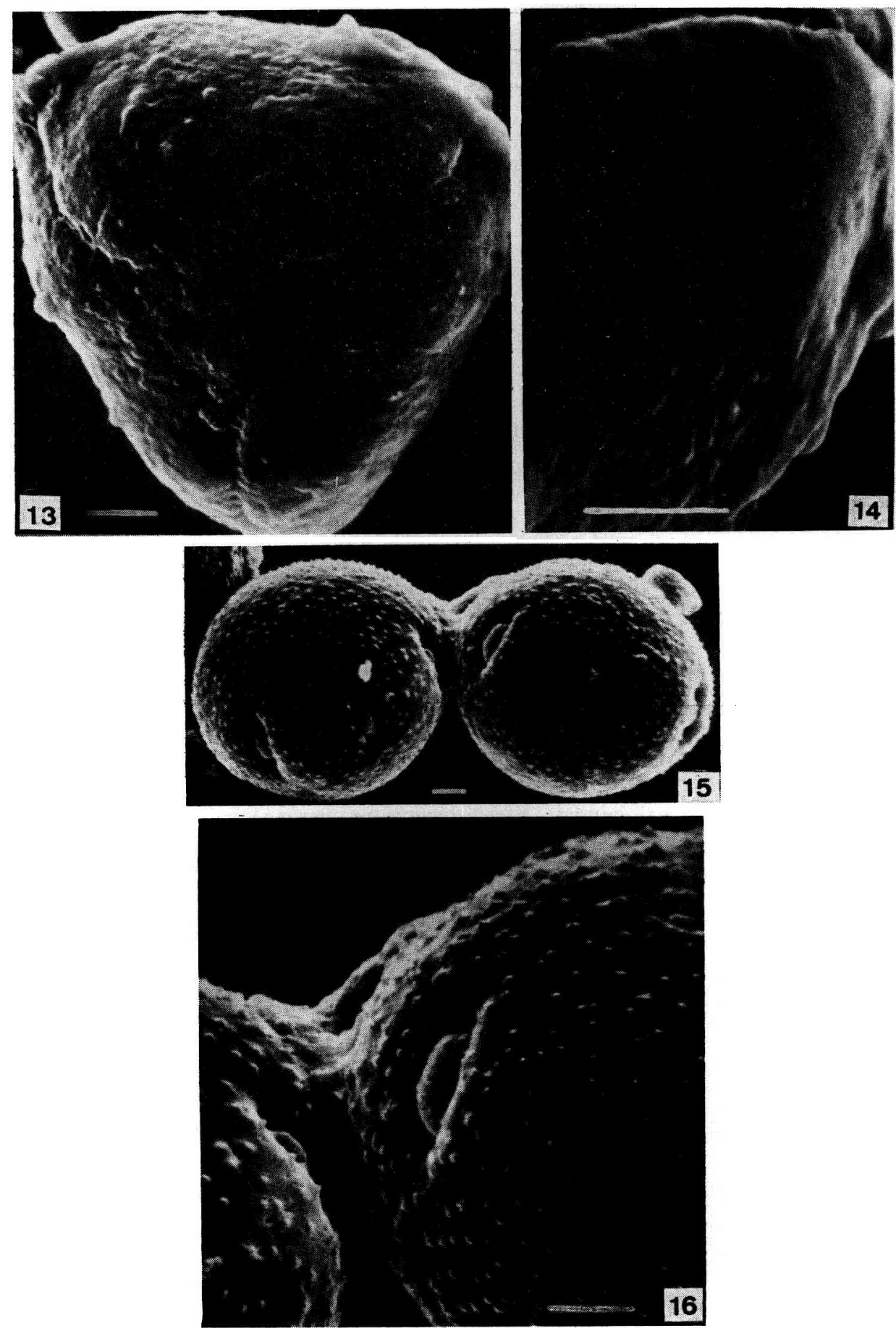

Ortrud Monika Barth 
PLANCHE IV.

Le trait clair correspond à $2 \mu$.

Fig. 17 à 20. - Hyptis umbrosa

Fig. 21. - Vernonia scorpioides

TAFEL IV.

Die weissen Segmente in den Figuren entsprechen stets $2 \mu$.

Fig. 17-20. - Hyptis umbrosa

Fic. 21. - Vernonia scorpioides 

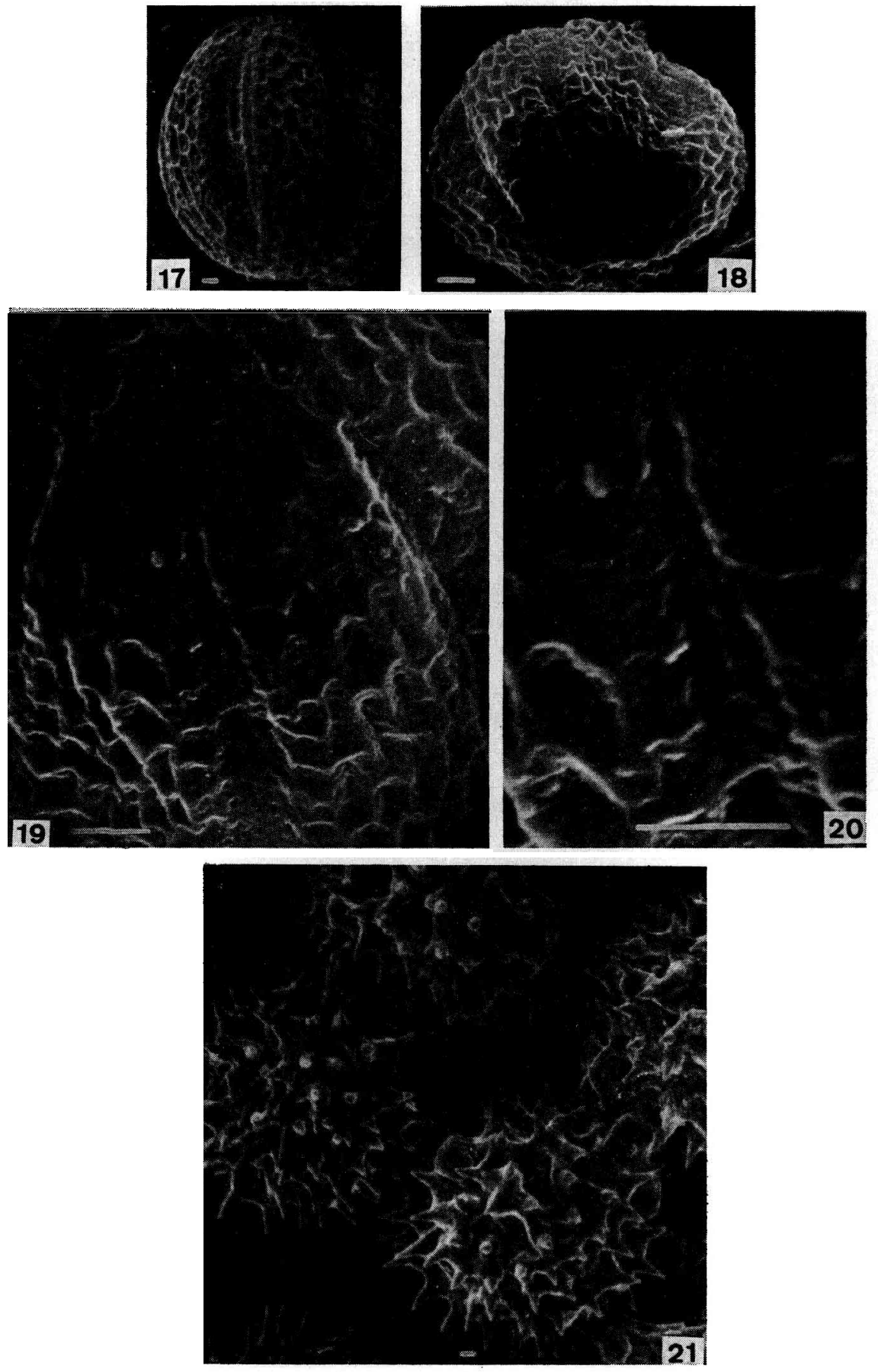

Ortrud Monika Barti 
